\title{
Una fortaleza convertida en archivo de la Corona: Simancas en el siglo XVI, nuevos datos para su estudio*
}

\author{
Eva Guerrero de Llanos \\ Universidad Complutense de Madrid \\ Departamento de Historia de Arte II (Moderno) \\ evaguerrerodellanos@gmail.com
}

Recibido: 25-02-2014

Aceptado: 09-07-2014

\section{RESUMEN}

Aportación de fuentes primarias inéditas que reconstruyen la historia del Archivo General de Simancas a mediados del siglo XVI, en el momento de su creación por parte de Carlos V y el secretario Francisco de los Cobos, alcaide de la fortaleza. Se dan nuevas noticias sobre las reformas llevadas a cabo en la época que constituyen el germen del actual archivo, y que siguen un memorial de Luis de Vega, Maestro Mayor de las Obras de su Magestad.

Palabras clave: Simancas, archivo, fortaleza, arquitectura, Valladolid, Renacimiento, Francisco de los Cobos, Juan Mosquera de Molina, Hernando Bernaldo, Luis de Vega, Francisco de Salamanca, Rodrigo de la Riva.

\section{A fortress converted into a Monarchy's Archive: Simancas sixteenth century, new data for study}

\begin{abstract}
Contribution of primary sources for the history of the General Archive of Simancas in the mid-sixteenth century, at the time of its creation by Carlos V and secretary Francisco de los Cobos, commandant of the fortress. The article gives new information about the reforms made in that period which are the seeds of the current archive, and following a memorial of Luis de Vega, Architect of his Majesty.
\end{abstract}

Key words: Simancas, archive, fortress, architecture, Valladolid, Renaissance, Francisco de los Cobos, Juan Mosquera de Molina, Hernando Bernaldo, Luis de Vega, Francisco de Salamanca, Rodrigo de la Riva.

\footnotetext{
* Este trabajo es resultado del Proyecto de Investigación I+D "Los Lugares del Arte, del taller del artista al espacio expandido en la sala de esposición” HAR2010-19406 y de la Beca FPI-UCM otorgada por la Universidad Complutense de Madrid en el año 2010.
} 


\section{El archivo como pieza esencial del estado moderno}

El Archivo de Simancas es, sin lugar a dudas, uno de los más importantes del mundo pero "lo transcendental del Archivo de Simancas no es su creación ni su existencia si no la conciencia de su necesidad" ${ }^{1}$ ya que el gérmen de esta institución constituye un hito en el mundo moderno castellano, entendiendo esa modernidad en el entorno de una administración y un gobierno que aún en el siglo XVI, estaba atrasado y disperso. No se concibe una administración moderna sin un archivo, por ello su creación supone un paso adelante en la Monarquía Hispánica, ya que se trata del nacimiento del sistema archivístico en el cual se establece que los documentos que ya no se necesitan en las oficinas de los organismos de la organización central, deben pasar a englobar el Archivo de Simancas, para su conservación perpétua y sobre todo, como instrumentos indispensables del gobierno eficaz de los reinos. A todo ello se suma que en la actualidad, la envergadura y alcance de sus fondos lo hacen imprescindible sobre todo para el estudio de la Historia Moderna, ya que aloja toda la documentación generada por los organismos de la administración central primero de los Austrias, y luego de los Borbones (en algunos casos hasta mediados del siglo XIX), revelando la estructura de sus fondos el esqueleto administrativo donde nació cada documento. Asímismo, el archivo destaca por el edificio que lo alberga, de una importancia histórica a la que se añade el valor de ser pionero en su género, y cuya transformación para tal fin trataremos más adelante.

Históricamente la Corona de Castilla carecía de un depósito documental a diferencia de sus reinos vecinos, que habían trabajado en ello con anterioridad. En Aragón hay indicios históricos de un archivo condal desde mediados del siglo IX aunque la mención más antigua que se conserva del Archivo de la Corona de Aragón es del 25 de octubre de $1180^{2}$ y su configuración plena, tras dos siglos de custodia de documentación, se llevará a cabo en 1346 con el nombramento del primer archivero. En el caso de Portugal el archivo acompañaba a los monarcas en su itinerancia por el reino, pero se tiene noticia de que entre 1352 y 1378, fechas parecidas a las que se han citado para el nombramiento de primer archivero en Aragón, se escoge la torre principal del Castillo de Lisboa para su definitiva instalación. Esta torre pasará a denominarse hasta la actualidad Torre do Tombo, por alojar los libros del catastro y tombo de los bienes reales portugueses ${ }^{3}$.

1 RODRÍGUEZ DE DIEGO, J.L., "La formación del archivo de Simancas en el siglo XVI. Función y orden interno", en: El Libro Antiguo Español, Coleccionismo y Bibliotecas (Siglos XV-XVIII), T. IV. Salamanca, Ediciones Universidad de Salamanca-Patrimonio Nacional-Sociedad Española de Historia del Libro, 1998, p. 520 .

2 Es muy interesante desarrollo durante los reinados posteriores, la historia completa del Archivo de la Corona de Aragón en: UNDINA MARTORELL, F., Guía Histórica del Archivo de la Corona de Aragón, Madrid, 1986.

3 Desde el reinado de Joao I (1357-1433) existe el cargo de "guarda-mor do reino" equivalente al de archivero, y en 1526 se llega a la madurez con la redacción del primer regimiento o reglamento del archivo a cargo de Tomé Lopes. PEDRUELO, E., "El Archivo General de Simancas. De archivo real a archivo público", en: Valladolid, ciudad de archivos. Valladolid, Universidad de Valladolid, 2011, p. 38. 
Es posible que el nomadismo de la corte castellana y la preocupación de la guerra contra los musulmanes, problema exclusivo de Castilla desde el siglo XIII, sean dos de los principales motivos por lo que se restó importancia a la creación de un archivo donde depositar todos los documentos gestados por la administración central. No obstante, una vez acabados los conflictos, resulta extraño ver cómo los Reyes Católicos, que legislaban de forma reiterada sobre cuestiones como la conservación de los protocolos notariales, la documentación municipal, la Chancillería y Registro de Corte o sobre la forma de expedición de determinados documentos, no traten de llevar a cabo una forma de conservar los papeles del Estado siguiendo el ejemplo de Aragón, reino del que también eran soberanos ${ }^{4}$.

Cuando se habla de los precedentes del Archivo de Simancas, es común remontarnos a la figura de Juan II y Enrique IV ${ }^{5}$ ya que la mayor parte de los historiadores remiten a una afirmación que hizo Agustín Riol a principios del siglo XVIII en su Informe sobre los archivos españoles y el desorden que en ellos había, escrito para Felipe V: "Don Juan II y Don Enrique IV mandaron recoger algunos en el castillo de la Mota de Medina y en el Alcázar de Segovia"“. Según explica Ángel de la Plaza, en el castilo de la Mota de Medina del Campo se custodiaron una serie de documentos, al igual que en Segovia, pero parece probable que fuera en época de los Reyes Católicos y no antes, sobre todo por las fechas de los fondos que allí se hallaron, datados en 1480 y años posteriores. ${ }^{\text {? }}$

Documentalmente se ha podido consignar que los primeros indicios de archivos en Castilla se llevan a cabo en época de Isabel y Fernando. Por una pragmática fechada en Medina del Campo a 24 de Marzo de $1489^{8}$ se decreta que en la casa donde estuviese alojada la Chancillería en Valladolid hubiera una cámara en la que se debían guardar documentos de carácter judicial tales como pleitos, pragmáticas o privilegios relativos a la Corte, creándose así el primer archivo de Castilla, el de la Audiencia y Chancillería ${ }^{9}$. Esta misma disposición se aplicó por medio de otra pragmática fechada a 20 de Noviembre de 1494 a la nueva Chancillería creada en Ciudad Real, que posteriormente se trasladaría a Granada ${ }^{10}$. Además, en las ordenanzas de Alcalá de Henares fechadas a 24 de Febrero de 1498 se ordena al registrador mayor ${ }^{11}$ que lleve a la Chancillería los registros con más de tres años de antigüedad junto con los anteriores a 1489, orden que volverá a repetirse en 1502 y que será una constante hasta

4 DE LA PLAZA BORES, A., Archivo General de Simancas. Guia del Investigador. Madrid, Dirección General de Bellas Artes, Archivos y Bibliotecas. Subdirección general de archivos, 1980

5 ROMERO DE CASTILLA Y PEDROSO, F., Apuntes históricos sobre el archivo general de Simancas. Madrid,Imprenta y estereotipia de Aribau y Compañía, 1873, p. 7.

6 RIOL, A., Informe que hizo el Secretario D. Santiago Agustín Riol al Rey D. Phelipe V. Manuscrito, Biblioteca Nacional de España, 1701, p. 79.

7 DE LA PLAZA BORES, A., op.cit., p. 24.

8 AGS, CÁMARA DE CASTILLA (en adelante CCA), DIVERSOS DE CASTILLA, LEG. 1, FOL. 66.

9 Sobre el Archivo de la Chancillería y su origen: MARTÍN POSTIGO, M.S., Historia del Archivo de la Real Chancillería de Valladolid, Valladolid, 1979.

10 ROMERO DE CASTILLA Y PEDROSO, F., op.cit, p. 8.

11 El Registrador era un empleado que tenía a su cargo el registro de todas las cartas y provisiones emanadas del Rey. Debía residir en la corte, seguirla y llevar siempre consigo el Registro. 
finales de siglo, cuando en 1592 Felipe II ordena que toda esta documentación pase al ya formado Archivo de Simancas. ${ }^{12}$

Los Reyes Católicos, en su campaña de ordenación de la documentación del Reino en estas fechas, realizaron otros cambios que sin duda, serían un precedente claro de la creación del Archivo de Simancas. En Sevilla a 9 de Junio de 1500 se dio una ordenanza por la que los corregidores estaban obligados a hacer construir un arca con tres llaves para depositar en ella los privilegios y papeles del concejo, disponiendo además que el escribano del municipio tuviese un libro en el que se copiaran todos los privilegios y sentencias dadas en negocios de la villa, y en otro registro se transcribiesen las provisiones y cédulas emanadas por los Monarcas. Del mismo modo, en 1501 se publicó una nueva pragmática por la que los escribanos de todos los lugares del reino debían formar un registro donde transcribiesen las cartas y ordenanzas enviadas por los Monarcas a cada localidad, seguida ésta de disposiciones en 1502 y 1503 para la custodia y conservación de los procesos seguidos en la audiencias y tribunales del reino y de los que obraban en las escribanías públicas y de cámara. ${ }^{13}$

Así pues, es el reinado de los Reyes Católicos el punto de partida para el estudio de la ordenación de los documentos en España siendo el verdadero precedente de Simancas el archivo que manda organizar Fernando el Católico en Valladolid, por Real Provisión de 23 de Junio de $1509^{14}$. En esta fecha se designa al Bachiller Salmerón para que recogiese los papeles dispersos tocantes a la corona y los depositase en un archivo "que yo mando hazer en la villa de Valladolid en la casa de audiencia e Chancilleria" ${ }^{15}$. Este proyecto no llegó a buen puerto ya que la labor de Salmerón no sólo era recoger, custodiar y sistematizar los documentos sino hacer dos copias de los que se consideraran más importantes, y aunque hay constancia documental de que el Monarca deseaba que la labor se llevara a cabo ${ }^{16}$ el propósito quizá por demasiado ambicioso o complejo, solo tuvo un principio de realización.

$\mathrm{Y}$ aunque siguiendo este hilo cronológico, Francisco Romero de Castilla atribuye la primera idea de formar un archivo en la fortaleza de Simancas al Cardenal Cisneros por una carta fechada a 12 de Abril de 1516 en la que ordena la recogida de papeles a los secretarios, receptores, oficiales y escribanos de cámara, no parece que esta idea cobrara forma ${ }^{17}$ ya que en la citada carta ni siquiera se menciona dicha fortaleza y no es hasta el reinado de Carlos I cuando la idea de un archivo en la villa de Simancas empieza a cobrar forma y se lleva a cabo. En 1531, demostrando una conciencia clara de creación de un archivo o al menos, de ordenación de las escrituras, Carlos I obtiene una bula papal por la cual todo el que tuviera en su posesión papeles de interés general debía devolverlos bajo pena de excomunión. Esta bula es sin duda,

12 PEDRUELO, E., op.cit, p. 39.

13 ROMERO DE CASTILLA Y PEDROSO, F., op. Cit, p. 9.

14 AGS, QUITACIONES DE CORTE, LEG. 12, BACHILLER SALMERÓN, apud DE LA PLAZA BORES, A., op. cit., p. 24; RODRÍGUEZ DE DIEGO, J.L., Instrucción para el gobierno del Archivo de Simancas (Año 1588). Ministerio de Educación y Cultura, Leganés, 1988, p. 29.

15 DE LA PLAZA BORES, A., op. cit., p. 24.

16 RODRÍGUEZ DE DIEGO, J.L., op. cit., p. 29. El autor recopila diferentes órdenes enviadas por el Monarca a Salmerón para la recopilación de papeles de Juan Velázquez, contador mayor.

17 ROMERO DE CASTILLA Y PEDROSO, F., op. Cit, p. 19. 
el germen del archivo que diez años después se crearía en la fortaleza de Simancas. El monarca, y sobre todo su secretario Francisco de los Cobos responsable absoluto de su creación, al percatarse del desorden existente en los documentos relativos a la corona, comenzaron a desarrollar la idea de crear un archivo donde alojar toda la documentación que en ese momento estaba esparcida por el territorio español en manos de la nobleza, secretarios, escribanos o receptores, entre otros muchos, o en lugares como el Castillo de la Mota, el Alcázar de Segovia, el Convento de San Francisco en Burgos o el de San Benito en Valladolid, sin contar con los ya archivados en la Chancillería de la misma ciudad ${ }^{18}$, y que era necesario centralizar, preservar y ordenar.

\section{Francisco de los Cobos. La creación de un archivo en la fortaleza de Simancas}

Francisco de los Cobos y Molina, nacido en la localidad gienense de Úbeda, donde tanto la rama familiar como su propia figura son esenciales por los ejemplos de patronazgo arquitectónico que se conservan en la villa es, sin lugar a dudas, una de las personalidades más influyentes de la época y personaje clave para comprender algunas de las vicisitudes del reinado de Carlos I de España. Caballero de Santiago, Comendador Mayor de León, Adelantado de Cazorla, Contador Mayor de Castilla, Secretario de Estado del emperador Carlos I, Señor de Sabiote, Jimena, Recena, Torres, Canena y Vellisca, y además Alcaide de la Fortaleza de Simancas, es el verdadero responsable de la fundación del Archivo General de Simancas y sobre todo, de su gestación.

La inexplicable falta de orden en la documentación castellana culmina en el año 1540 según parece, gracias al secretario imperial. Se conserva una minuta de carta de Francisco de los Cobos al secretario Juan Vazquez de Molina fechada a 26 de Junio, en la que aparece por primera vez la fortaleza de Simancas como lugar adecuado para conservar la documentación emitida por la administración y por el propio Monarca ${ }^{19}$ en la que explica "según me han dicho las escripturas que tenia el licenciado Acuña con el titulo de archivio me dizen que no estan en el recaudo que conbiene, ay va una cedula en blanco para que se entregen. Consultareis señor con S.M en quien quiere que se pongan, entretanto se ordena lo del archivo que este verano mandare a dar la orden de que se haga en Simancas y mirad si entre tanto están bien en poder de Mosquera o de quien pareciere "20, una idea que comenzó a gestarse en el año 1539, cuando el propio Cobos escribe a su sobrino Juan Mosquera de Molina una carta fechada en Madrid a 29 de Octubre indicándole una serie de reformas que había que hacer en el edificio, seguramente con el fín de convertirla en archivo un año después ${ }^{21}$. Asímismo, el 24 de Febrero del año siguiente, meses antes de la citada minuta, se halla

18 VVAA., Guía del Archivo General de Simancas. Valencia, Dirección General de Archivos y Bibliotecas, 1958 , p. 6.

19 AGS, ESTADO, LEG. 49, FOL. 81, apud DÍAZ SÁNCHEZ, F., Guía de la villa y el archivo de Simancas. Madrid, Tipografía de Manuel G. Hernández, 1885, p. 19.

20 Juan Mosquera de Molina era el teniente de alcaide de la fortaleza de Simancas en esa fecha.

21 AGS, CONTADURÍA MAYOR DE CUENTAS (en adelante CMC), 1EP, 1410. 
una carta del mismo Francisco de los Cobos dirigida a Hernaldo Bernaldo, su mayordomo y teniente de alcaide también a su vez, de la fortaleza, en la que vuelve a tratar las reformas que debían hacerse en la misma durante los meses sucesivos, haciendo referencia a un memorial llevado a cabo por el que ya había sido nombrado Maestro Mayor de las Obras de su Magestad, Luis de Vega ${ }^{22}$.

Con la idea de creación del archivo en marcha, a 16 de Septiembre se emite en Bruselas una Cédula Real, la primera orden oficial para la recogida de documentación en la fortaleza vallisoletana ${ }^{23}$. Este documento está dirigido a todas las personas que poseyeran "escripturas de qualquier calidad e importancia" que hubiera tenido a su cargo el Licenciado Acuña, ya fallecido. Papeles que iban a formar parte del archivo que Francisco de los Cobos quería crear y que tenían que entregarse con un inventario adjunto a Juan Mosquera de Molina, alcaide de la fortaleza de Simancas.

La recogida se pone en marcha por tanto a principios de la década de los cuarenta, y está apoyada por una serie de documentos oficiales emitidos por el propio monarca o por su hijo Felipe en los que se piden los papeles que conformarán el grueso del archivo a diferentes organismos o personas. De este modo, en 19 de Febrero del año 1543, Carlos I por Real Cédula pide al regente y oidores de la Audiencia y Chancillería de Valladolid la documentación del Castillo de la Mota y de Medina del Campo para que sea entregada en el archivo de la villa de Simancas ${ }^{24}$, y un año después es el príncipe Felipe quien reclama los papeles pertenecientes a Indias, que serían guardados en un arca con dos llaves ${ }^{25}$ conservado en la actualidad en el propio Archivo General de Simancas.

Sin embargo, la mayor parte de la documentación relativa a la Corona de Castilla o a los asuntos de Estado no estaba recaudada en estos lugares, sino que se encontraba dispersa en manos de diferentes personalidades. Es por ello que en 1545, se lleva a cabo una labor de recogida que parte de Reales Cédulas, en un alto porcentaje firmadas por el príncipe Felipe y en las que pide la entrega de los documentos para el archivo simanquino a personas en cuyo poder hubiera escritos tocantes a la corona y patrimonio real como Presidentes del Consejo Real y Chancillerías de Valladolid y

22 Ibidem. Las referencias a las labores de reforma de la fortaleza para su transformación en archivo serán tratadas en profundidad en este mismo artículo.

23 DE LA PLAZA BORES, A., op. cit, p. 26; AGS, CCA, LEG. 247.

24 Transcribimos debido a la relevancia del documento. "Sabed que, a la demanda del licenciado Pedrosa nuestro fiscal en esta audiencia, os encargamos, como asi mismo el licenciado Oviedo nuestro fiscal tambien y a Agustín de Zarate nuestro escribano de Camara, de ir a la villa de Medina del Campo y examinar ciertas escrituras concernientes a nuestra corona y patrimonio real y a otras cosas, y hacernos relación de lo en ellas contenido y de la manera como estaban goardadas. Habiendo visto en nuestro consejo dicha relacion y el inventario de dichas escrituras, hemos resuelto sean trasladadas a la fortaleza de la Villa de Simancas, para que en esta esten mejor guardadas y puedan ser consultadas más facilmente por nuestros fiscales y por las personas que las hayan menester. Mandare al Conserge de la fortaleza de la Mota para que entregue las dichas escripturas a la persona comisionada por vos al efecto. (...) Se construira para guardarlas una arca con dos llaves: una quedara en poder del presidente de dicha Chancillería y la otra en manos del conserge, y es mi voluntad que, sin provision mia, no se pueda abrir la dicha arca ni sacara título alguno para nadie”. Publicado por ROMERO DE CASTILLA Y PEDROSSO, F., op. cit., p. 20.

25 El hábito de crear arcas con varias llaves era común en la época. Así se hizo, por ejemplo, en el Alcázar de Madrid. Existió un arca con tres llaves (para guardar el dinero vinculado a las obras) en poder del Veedor, del Mayordomo, y del Maestro Mayor. AGP, REGISTROS, CR, T. I, Fol. 236v. - 237r. 
Granada, Contadores Mayores de Cuentas, Contadores Mayores de Hacienda, Alcaldes mayores y Gobernador de Galicia, Corregidores o los herederos de figuras como Lope Conchillos y otros secretarios falecidos, o Don Juan Manuel, el Doctor Puebla, entre muchos embajadores en tiempo de los Reyes Católicos. ${ }^{26}$. Cédulas entre las que encontramos otras dirigidas a Juan Mosquera de Molina donde el Príncipe se interesa por el orden de los documentos, -lo que nos muestra una vez más la personalidad del que sería posteriormente rey Felipe II, atento a este tipo de cuestiones- ${ }^{27}$ y que culminan con una serie de cédulas sueltas, a medida que se iba conociendo qué personas podían tener documentos de interés para el archivo, entre ellas las dirigidas a María de Mendoza, viuda ya en esos años de Francisco de los Cobos, para pedirle el grueso de la documentación que el secretario imperial tenía en su poder cuando fallece en $1547^{28}$. Estos papeles de Cobos, sin duda, fueron una de las grandes aportaciones a los fondos del actual Archivo de Simancas.

Recogida alguna documentación de interés, y con las obras de las que hablaremos a continuación comenzadas, faltaba para completar la puesta en marcha del archivo, nombrar personas que se ocupasen de conservar, atesorar y colocar los papeles reunidos y de recoger la que iba llegando a la fortaleza, en poder de funcionarios e instituciones. A propuesta del secretario Francisco de los Cobos y del presidente del Consejo Real ${ }^{29}$ el Emperador nombra "Tenedor" del Archivo al licenciado Antonio Catalán, relator del Consejo, con 100.000 maravedís de salario, y al teniente de alcaide Juan Mosquera de Molina para que sirviera de apoyo y ayuda al Tenedor de las Escrituras, con 40.000 maravedís de quitación ${ }^{30}$.

Es este el momento en el que podemos considerar que el Archivo de Simancas comienza a cumplir su función, la que el secretario Francisco de los Cobos había planeado desde la década de los treinta de ese siglo XVI. En los subsiguientes decenios la recogida de documentación sigue su cauce, sobre todo durante el reinado de Felipe II, cuando se amplía para dar cabida a todos los papeles que llegan hasta

26 AGP, REGISTROS, CR, T. I, Fol. 2 - 9. Tres nutridas series de cédulas que ya fueron mencionadas por DE LA PLAZA BORES, A., op. cit., p. 28 y RODRÍGUEZ DE DIEGO, J.L., "La Formación...” (op. cit)., p. 527.

27 La importante reforma del Archivo General de Simancas que convierte la fortaleza en el edificio que hoy podemos ver se lleva a cabo durante su reinado.

28 AGP, REGISTROS, CR, T. I, Fol. 7-8, 10.

29 AGS, ESTADO, LEG. 56, FOL. 13, apud DE LA PLAZA BORES, A., op. cit., p. 27.

30 Minuta de Despacho en AGS, ESTADO, LEG. 21, FOL. 194, citada por DE LA PLAZA BORES, A., Op. cit, fol. 27. Nos interesa especialmente: “...entendido el poco recaudo cuydado y orden que siempre ha habido en la guarda y conservacion de las dichas scripturas y los daños y inconvenientes que dello se hanseguido y adelante se podrian seguir y querido poner remedio en ello como en cosa tan importante como servir a la corona real habiendoos acordado y mandado que se haga un archivo de las dichas ecripturas como ya se ha comenzado a hazer en la nuestra fortaleza de Simancas y que alli se recogan y junten todas las escripturas tocantes a mi patrimonio y corona real que estovieren en quales quiera partes de los dichos reinos y que a un letrado se de cargo dellas el qual tenga una llave de la rexa o puerta de la pieza o alhacena donde han de estar las dichas escripturas de cada genero de cosas y el alcaide o su theniente que fuere de la dicha fortaleza de Simancas otra y a quando se pusiere en el dicho archivo o sacare del alguna escriptura se hacen los dos presentes..." 
la fortaleza ${ }^{31}$ y se promulga uno de los textos más importantes para la historia de los archivos: las Instrucciones para el Gobierno del Archivo de Simancas, fechadas en $1588^{32}$. Estas Instrucciones se consideran en la actualidad el primer reglamento de archivos entendiendo como tal el conjunto de normas claras, precisas, que regulan de una forma coherente y duradera las funciones integrantes de la vida de un archivo que no son otras que las que se atribuyen modernamente a la archivística, es decir: reunir, conservar, describir y distribuir. Aunque en épocas anteriores se habían establecido otras disposiciones sobre archivos ${ }^{33}$ tocantes a diferentes aspectos de los mismos, este escrito, de treinta capítulos, es novedoso e irrepetible en tanto en cuanto se trata de unas ordenanzas sobre el funcionamiento de la institución, minuciosas y reguladoras de las bases documentales de producción, guarda y organización y que fueron publicadas sin límites temporales. Estas ordenanzas, el atesoramiento de documentación comenzado en la década de los cuarenta del siglo XVI, la función de los archiveros que trabajaron en el organismo desde su creación ${ }^{34}$ y las reformas de la fortaleza llevadas a cabo sobre todo, en el siglo XVI, convirtieron el archivo en uno de los más relevantes e imprescindibles, actualmente y a nivel mundial, para el estudio de la época de los Austrias.

\section{Una fortaleza convertida en archivo. El castillo de Simancas y su reforma durante el reinado de Carlos V}

Si hay algo que impresiona cuando el investigador acude a Simancas, es el edificio en el que está alojado el archivo. La gran fortaleza se recorta sobre el cielo vallisoletano y da una solemne bienvenida acorde con la documentación que se va a consultar ${ }^{35}$. Pero este gran edificio no fue construído para tal fin, sino que se fue adecuando a él con el paso de las décadas comenzando por la primera reforma en los años cuarenta del siglo XVI, que nos ocupa en el presente estudio y que ha sido hasta el momento, casi pasada por alto.

31 Un ejemplo de que las peticiones de documentación continúan es: ARChV, CÉDULAS Y PRAGMÁTICAS, CAJA 0003.0031, sobre el traslado de documentación a Simancas desde el Monasterio de San Benito en Valladolid entre 1562 y 1578.

32 RODRÍGUEZ DE DIEGO, J.L., Instrucción para el gobierno del Archivo de Simancas (Año 1588). Madrid, Ministerio de Educación y Cultura, Dirección General del Libro, Archivos y Bibliotecas, 1998. El autor hace un estudio muy interesante sobre el texto original, con una reproducción en facsímil que transcribe íntegramente.

33 CASANOVA, E., Archivística. Siena, Lazzeri, 1928.

34 Destacamos la reconocida y eficiente dirección del archivo por parte de Diego de Ayala (1563 - 1588), primer archivero "real" de Simancas, cuya gestión supone la base para los posteriores archiveros y hasta la actualidad. Durante su dirección el archivo crece tanto en el volúmen de sus fondos como en lo tocante al propio edificio, que sufre una gran reforma muy controlada por Ayala. Sobre Diego de Ayala y la gestión del archivo es interesante el capítulo que le dedica DE LA PLAZA BORES, A., op. cit. p. 31 - 46.

35 Fig. 1 . 


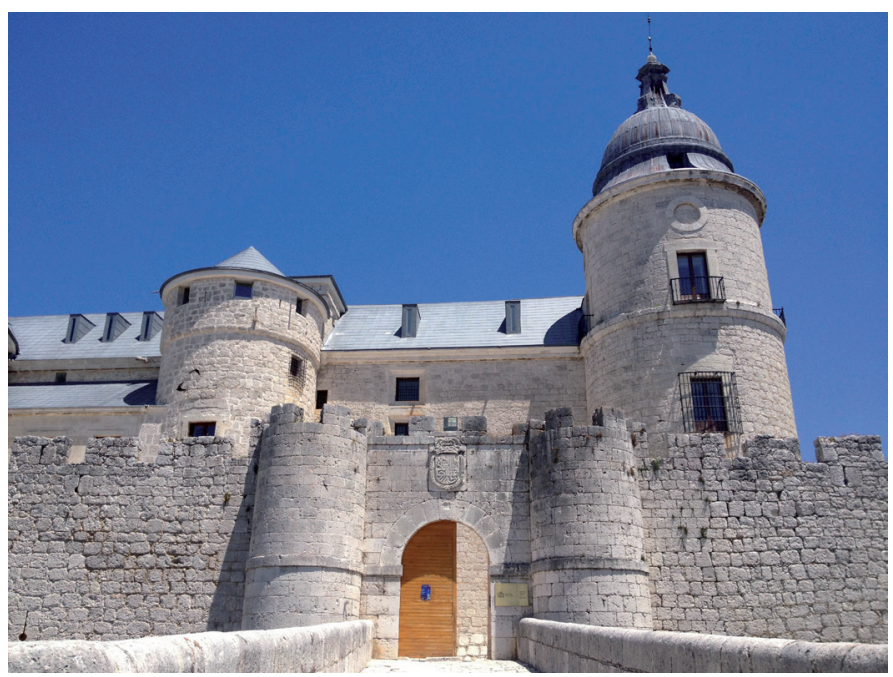

Fig. 1. Fachada del Archivo General de Simancas - Entrada Principal. Fotografía de la autora. Insertar acorde con su referencia, en la nota al pie 35.

La fortaleza, que Vicente Lampérez se apresuraba a fechar "indudablemente en los siglos X y XI" aunque añadiendo "pero las fábricas que hoy vemos no son tan antiguas" 36 , data del siglo XV, y pertenecía al Almirante Don Alonso Enríquez.

En las Antiguedades y sucesos memorables sucedidos en esta muy noble y muy antigua villa de Simancas, escrito por el clérigo simanquino Antonio Cabezudo en el siglo XVI ${ }^{37}$ dice que existía "un castillo alto con una gran torre" a la Puerta de Mirabete que al parecer, era de tierra y argamasa gruesa, y que de esta fortaleza habla el cronista Diego Enríquez del Castillo en su Crónica de Enrique IV, "cuando la tomó el Almirante D. Fadrique Enrriquez, que fue padre de D. Alonso, el que después la derribó para edificar la suya, que hizo de nuevo desde los cimientos de cantería labrada que ahora vemos". Lo que nos quiere decir Antonio Cabezudo, en otras palabras, es que a finales del siglo XV existía una edificación de la que se apoderó en septiembre de 1467 el Almirante Don Fadrique y que fue derribada por su hijo y sucesor en el almirantazgo Don Alonso Enriquez (1473 - 1485). Fue él quien levantó la fortaleza de cantería que vería Cabezudo en 1580 y que en la actualidad existe -salvando las reformas acometidas-, convertida en archivo. ${ }^{38}$

Ángel de la Plaza, en su espléndida introducción a la Guía del Investigador, aporta una serie de documentos que refuerzan esta hipótesis, quedando indudablemente

36 LAMPEREZ Y ROMEA, V., Arquitectura Civil Española. Tomo I. Madrid, Saturnino Calleja, 1922, p. 265.

37 Hoy podemos encontrarlo íntegro en: CABEZUDO, A., Antiguedades y sucesos memorables sucedidos en esta muy noble y muy antigua villa de Simancas, por Don Antonio Cabezudo, cura de la parroquia de la misma villa, beneficiado de Preste, 1580. Transcrito por Teresa Salvador Berrueco. Valladolid, Diputación Provincial de Valladolid, 2004.

38 DE LA PLAZA BORES, A., Op. cit., p. 15. 
clarificada. La ciudad de Valladolid, en una exposición a Felipe II de sus derechos jurisdiccionales sobre la villa de Simancas dice, aunque sin precisar fecha ni persona: "Subcedio que en tiempo que en ellos (los reinos) no auia paz y justicia de que por la bondad de dios en tiempo de Vra. Magestad gozan el que a la sazon hera Almirante de Castilla ocupo aquel lugar (Simancas) lo començo a llamar villa y labro una fortaleza para poderlo mejor sustentar" ${ }^{39}$. Además cita un Privilegio fechado en Simancas a 2 de Febrero de 1474, por el cual el Almirante Don Alonso Enriquez concede varias mercedes y exenciones a su villa de Medina de Rioseco en atención por los servicios prestados "y señaladamente haviendo respecto al socorro e seruicio que de vuestra mera voluntad me fesistes de vn quento de maravedís pagados en cinco años primeros siguientes para con que se labre e edefique e sea labrada e edeficada la fortalesa e castillo de la villa de Symancas lo qual era e es a mi cosa muy necesaria e vtile e prouechosa" 40 .

La fortaleza, por lo tanto, se construye en el período limitado entre septiembre de 1467, cuando el Almirante Don Fadrique se establece en la villa de Simancas, y el 15 de septiembre de 1480, momento en el que su hijo Don Enrique cede la fortaleza a los Reyes Católicos. Parece, no obstante, que la fortaleza fue construída por el segundo según dice Antonio Cabezudo y cedida practicamente al ser culminada ${ }^{41}$. La cesión del edificio y de sus derechos jurisdiccionales se llevó a cabo bajo la promesa de la entrega de "cierto número de vasallos con cierta renta" al Almirante, que al no poder ser entregados, se acordó el pago de 300.000 maravedís de juro de heredad y 900.000 al quitar, a 10.000 el millar, una retribución que se dispone finalmente el 15 de febrero de 1490 con un albalá en el que se ordena la expedición de los privilegios de juro a favor de Don Fadrique. La fortaleza de Simancas queda por tanto en posesión de los Reyes Católicos, desde el mes de marzo de $1490^{42}$.

Hasta nosotros ha llegado parte de aquella fortaleza de los Almirantes de Castilla que posteriormente fue guardajoyas ${ }^{43}$ y prisión $^{44}$, con una reforma que comienza a mediados del siglo XVI, y otras reformas sucesivas mucho más reconocidas por la historiografía, sobre todo las que se llevan a cabo durante el reinado de Felipe II $^{45}$.

\footnotetext{
39 AGS, ESTADO, LEG. 129, FOL. 304, apud Ibidem., p. 16.

40 Ibidem. El original de este privilegio se conserva, según el autor, en el Archivo Municipal de Medina de Rioseco, pero no aporta datos archivísticos.

41 Existen diversas leyendas sobre la cesión de la fortaleza a los Reyes Católicos. Es curiosa la que cuenta DEL PUlGAR, H., Crónica de los señores Reyes Católicos. Valencia, Imprenta de Benito Monfort, 1780, p. 173-174. En ella, la Reina Isabel había ido sola a buscar a Don Fadrique a la fortaleza de Simancas para someterle a su justicia, y al no encontrarlo allí ordenó a su padre, le entregara el edificio.

42 DE LA PLAZA BORES, A., Op. cit., p. 18. Este hecho queda ratificado por un albalá que se conserva en los libros de Tenencias de Fortaleza del Archivo General de Simancas (AGS, ESCRIBANÍA MAYOR DE RENTAS, TENENCIAS DE FORTALEZA, LEG. 4, Simancas) en el que se manda asentar al Comendador Pedro de Rivera 150.000 maravedís por la tenencia de la fortaleza de Simancas, que se le pagarían desde el 1 de marzo de 1490.

43 ÁlVAREZ PIÑEDO, F.J., y RODRÍGUEZ DE DIEGO, J.L., Los archivos españoles: Simancas. Madrid/Barcelona, Ludwerg Editores y Ministerio de Cultura, 1993, p. 20.

44 AGS, CCA, CÉDULAS, FOL. 130. Cédula Real fechada a 19 de Julio de 1527, dirigida a Juan de Vega, alcaide de la fortaleza, en relación a Domingo de Castro, preso en la misma.

45 Sobre las reformas ejecutadas durante el reinado de Felipe II, es muy interesante la obra de BUSTAMANTE GARCÍA, A., La arquitectura clasicista del foco vallisoletano (1561-1640). Valladolid, Institución Cultural Simancas, 1983, p. 160-167.
} 
La entrada de documentación en la fortaleza de Simancas en los años cuarenta del siglo XVI, y la idea -parece que unos años más joven- de Francisco de los Cobos, de convertir el edificio en archivo de la Corona exigía obras de reforma convenientes para el acomodamiento de estos papeles. La historiografía hasta la actualidad ha seguido la tesis que expresa cómo, en el momento de llevar la documentación a Simancas y archivarla, se elige una ubicación muy concreta: el torreón de Obras y Bosques, también llamado de Patronato Real o cubo de Carlos V. Las obras de reforma necesarias habían comenzado en otras partes de la fortaleza anteriormente, y habían sido encargadas por Cobos. Así aparece consignado en una carta que escribe el secretario imperial a su mayordomo Hernaldo Bernaldo fechada en Madrid a 29 de Octubre de 1539 sobre unas obras que se están llevando a cabo en la fortaleza y que al parecer, se basan en la colocación de nuevas puertas, ventanas y garitas, por valor de 8.000 maravedís y que han de ser terminadas antes del 10 de Noviembre de dicho año. No obstante, hace referencia en la propia misiva a "las otras cosas y reparos que vimos en la tasacion" con las que se refiere, seguramente, a la siguiente carta en la que se tratan las reformas destinadas al propio archivo en el Cubo de Obras y Bosques ${ }^{46} \mathrm{y}$ a otras obras de mayor envergadura hasta ahora desconocidas y que sólo podemos entender al adentrarnos en las cuentas de Hernando Bernaldo y del Bachiller Carrasco fechadas entre 1540 y $1545^{47}$. Esta documentación sin embargo demuestra, como se ha citado con anterioridad, que Cobos tenía en mente la creación del archivo y ya desde finales de la década de los treinta comienza con las reformas para adecuar la fortaleza a su nuevo cometido.

Con este fin, Francisco de los Cobos vuelve a escribir a su mayordomo Hernaldo Bernaldo una carta fechada en Madrid, a 24 de Febrero de 1540, donde explica las reformas que deben hacerse. Esta misiva, que debía ir acompañada por otra a Juan Mosquera de Molina quizá más amplia y que no ha sido hallada, relata el comienzo de la construcción del futuro archivo. Las obras deben seguir las pautas de un memorial escrito por Luis de Vega, Maestro Mayor de las Obras de su Magestad ${ }^{48}$, citado y descrito por Cobos en la carta. Los reparos mencionados en la misiva pasaban por una chapadura exterior de cantería, solar la casa, empedrar el patio y reformar las chimeneas, y todos ellos debían darse a destajo según las condiciones impuestas

46 AGS, CMC, 1EP, 1410, s.f. Documento íntegramente transcrito en el apéndice documental.

47 AGS, CMC, 1EP, 1318, 1339, 1380, 1410.

48 Luis de Vega ostenta el título oficialmente junto a Alonso de Covarrubias desde el año 1537, aunque tiene contacto con las obras reales desde 1528, cuando viaja a Granada para supervisar los planos del Palacio de Carlos V en La Alhambra, sin duda gracias al apoyo de su protector, Francisco de los Cobos. Para éste levanta, tras sus inicios como carpintero y maestro de casas en la ciudad de Alcalá de Henares, el palacio de la Corredera de San Pablo en la década de los veinte, en Valladolid, y reforma la casa-palacio propiedad de la familia en Úbeda (Jaén), a principios de los años treinta. Así mismo, hasta la muerte de Cobos en 1547 Luis de Vega tiene contacto permanente con él tanto en lo referente a las obras reales como en obras promocionadas por el propio secretario. No resulta extraño que el Comendador Mayor de León recurra a Vega para realizar un memorial de las obras que debían hacerse en la fortaleza de Simancas, una misión que es lógica para con su cargo como Maestro Mayor de Obras Reales y sobre todo, al recorrido que se documenta desde que ostenta el cargo, viajando incansablemente para hacer revisiones y modificaciones en dichas obras. La trayectoria de Luis de Vega, no obstante, se encuentra en revisión actualmente gracias a la tesis doctoral (en proceso) Arquitectura en la Corte de Carlos V: el Maestro Mayor Luis de Vega y su entorno. 
por el maestro Luis de Vega y con la máxima brevedad. Así mismo, Hernando Bernaldo y Juan Mosquera de Molina debían vigilar que todo se hiciera conforme a lo que escribía Cobos y Vega, con un presupuesto de ochocientos ducados que podían ascender cómo máximo a mil ${ }^{49}$. Las cuentas nos van a demostrar que estas sólo fueron las primeras pinceladas del gran lienzo que es la fortaleza de Simancas, que fue reformada a conciencia en estos años para cumplir perfectamente con el fin que se le había impuesto.

Aunque el memorial de Luis de Vega no se encuentra en ninguno de los libros de cuentas de Hernando Bernaldo, ni en otros posteriores, ni tampoco se ha hallado entre la documentación de Casa y Sitios Reales, Cámara de Castilla o Estado, con la carta de Francisco de los Cobos y sobre todo rastreando las cuentas, podemos hacernos una idea de la magnitud que comenzaban a cobrar las obras en la Fortaleza de Simancas. El secretario imperial había decidido crear el archivo y por ello acondicionaba, con el patrocinio regio, el edificio en el que se iban a depositar los papeles más importantes del reino. Obra de reformas que como decimos, comienza por los elementos más comunes (puertas, ventanas, chimeneas), pasa por los solados o empedrados de las zonas que se va a transitar, como es el caso, o por chapados de elementos que se encuentran en peor estado de conservación como era la parte exterior del edificio que actualmente se conserva practicamente sin cambios. Estas obras son el comienzo del Archivo General de Simancas y parece ser que sin descanso, una vez acabadas estas, se comienzan las de mayor envergadura: los cubos, el propio archivo, el corredor delantero del patio y las reformas en la Capilla y en el Cuarto Real. El importe de estas obras, llevadas a cabo entre 1541 y 1543, asciende a un millón ciento noventa y cinco mil quinientos maravedís ${ }^{50}$ se completarán los dos años siguientes, como veremos.

Según las cuentas que se encuentran archivadas en Simancas, en 1541 se trabaja en obras de reparos, sobre todo, de la zona exterior del edificio y además se reforman algunas tapias en mal estado, un puente de acceso a la fortaleza, la chapadura de la cava, una nueva chimenea que se construye debido al derrumbe de la anterior, y se colocan cerrojos, puertas principales y ventanas que sustituyen a las antiguas. ${ }^{51} \mathrm{El}$ edificio, una fortaleza del siglo anterior, estaba falto de algunos elementos necesarios para la labor que Francisco de los Cobos le había encomendado y estas necesidades no se centraron sólo los reparos citados, sino en otras de mayor envergadura que encontramos al seguir detalladamente las cuentas de los siguientes años.

49 Estos datos fueron citados sin ninguna referencia archivística por RODRÍGUEZ MARÍN, F., Guía histórica y descriptiva de los archivos, bibliotecas y museos arqueológicos de España que están a cargo del cuerpo facultativo del ramo. Madrid, Tipografía de la Revista de Archivos, Bibliotecas y Museos, 1916, p. 137. Esta publicación fue seguida por KENISTON, H., Francisco de los Cobos, Secretario de Carlos V. Madrid, Castalia, 1980, p. 264, igualmente sin aportar datos archivísticos. En un proceso de búsqueda exhaustiva en el Archivo General de Simancas se halló en primer lugar, una copia de la citada carta (AGS, CMC, 1EP, 1318, s.f) en la que se hace referencia a la original con una nota que dice "concertado con el escripto de la carta original firmada por el comendador mayor de leon hecha en madrid a veynte y quatro de henero año de mill y quinientos y quarenta. La original quedo en el otro libro". La carta original se encontró, definitivamente, en otro libro de cuentas de Hernando Bernaldo (AGS, CMC, 1EP, 1410, s.f).

50 AGS, CMC, 1EP, 1410. Reza así en el cargo de las obras descrito detalladamente por Hernando Bernaldo.

51 AGS, CMC, 1EP, 1318, 1410. 
En 1542 comienzan las referencias al archivo en las cuentas de Simancas aunque llaman poderosamente la atención otros gastos que se repiten de forma continua y de los que no se tiene constancia: los centrados en los tres cubos que se hacen en ese momento en la fortaleza con el fín de convertirse en archivo. Desde el mes de noviembre del citado año y hasta finales de 1543, aparecen de forma recurrente pagos a diferentes oficiales -albañiles, canteros, yeseros y finalmente carpinteros- por las obras de "los cubos que se hacen en la fortaleza", para, con el paso de los meses, especificar "el cubo postrero que se hace para el archivo" o "el cubo grande que se hace para el archivo". Además, por si el enorme cauce de pagos sobre los cubos no fuera clarificador, nos encontramos, para finalizar con la afirmación "monta la data de los tres cubos y archivos de la dicha fortaleza de Simancas ochocientos y noventa y cinco mill y ciento y treynta y dos mrs", a finales del año $1543^{52}$.

Las primeras cuentas que nos encontramos en relación al archivo se centran en la colocación de los documentos en el interior de un cubo que debía estar finalizándose - de construir o de reparar-, muy probablemente el situado al noroeste, que actualmente conocemos como "Cubo de Obras y Bosques o de Patronato Real". Se trata de la torre que la historiografía ha tenido por el único lugar de la fortaleza acondicionado en estas fechas como archivo, con dos salas en altura (en 1543 se construye una nueva aprovechando la plataforma del cubo, que se eleva mediante ladrillo). En la actualidad conserva la configuración creada en los años cuarenta del siglo XVI basada en una estructura envolvente de estanterías de pino perfectamente acondicionadas y cerradas para conservar los papeles, diseñadas en las dos alturas citadas, y divididas por una galería o andito ${ }^{53}$. De este modo, se paga en numerosas ocasiones a Antonio de Castro y otros carpinteros por trabajos en el archivo, vigas para cajones del archivo o por los propios cajones y marcos de los mismos. A su vez, nos encontramos pagos a otros carpinteros por cubrir el cubo que se hacía para el archivo, a canteros por las ventanas del mismo o datos curiosos como el hecho de que las ventanas se cubrieran con hojas de lata para que no entrara el agua de la lluvia y no se pudrieran los papeles ${ }^{54}$. De estos pagos podemos sacar en claro dos cosas: la primera de ellas es que, efectivamente, el Cubo de Obras y Bosques se acondicionó a la perfección para su nuevo fin de forma rápida y eficaz. El segundo hecho patente al revisar los pagos se centra en el propio cubo, que se encontraba en pésimas condiciones y tuvo que ser muy reformado para convertirse en el lugar principal de custodia de los documentos de la Corona. Por una carta fechada en Madrid a 26 de Enero de 1543 sabemos que la obra que ya se cita "del archivo" estaba acabada ${ }^{55}$. Los dos primeros pisos del cubo se culminan en esta fecha y aún hay doscientos ducados para la parte alta del mismo, en la que se trabaja meses después ${ }^{56}$. Francisco de los Cobos en esta misiva además hace

52 AGS, CMC, 1EP, 1410, s.f.

53 Fig. 2.

54 Así reza en las cuentas relativas al año 1542. Pagos a "Albaro del Pino, especiero, vecino de Valladolid doss myll e quarenta mrs por sesenta ojas de lata de marca mayor q dio las quales se gastaron en cubrir las ventanas de los archibos que salen al campo por q no se pudriesen del agua por libranza del dicho comendador mosquera fecha a diez de jullio de IVDXLII años"

55 Ibidem.

56 Fig. 3. 
hincapié en que "me paresce bien que se gasten en ella porque todo sera menester para las escripturas", lo cual nos muestra la relevancia que tenía para el secretario la obra del $\operatorname{archivo}^{57}$.

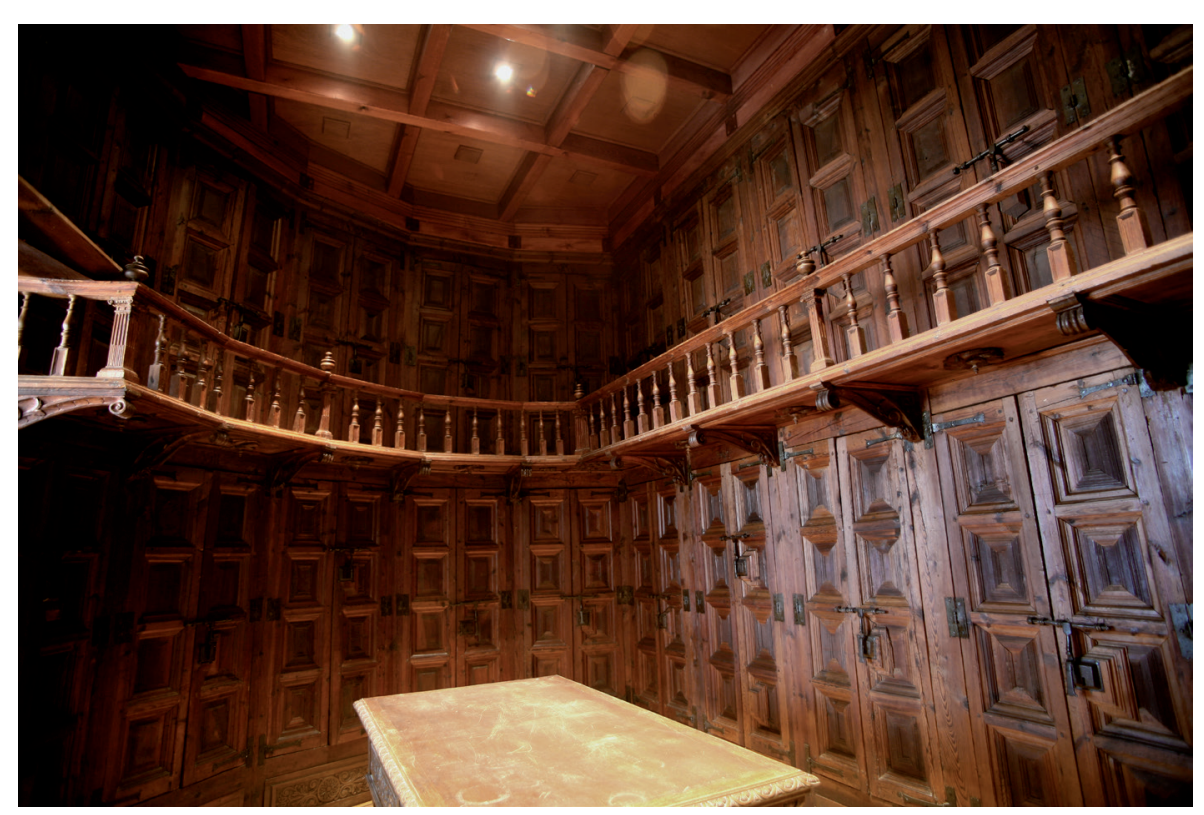

Fig. 2. Interior del Cubo de Obras y Bosques, Archivo General de Simancas, 1543. Fotografía propiedad del Ministerio de Cultura. Insertar acorde con su referencia, en la nota al pie 53.

Las cuentas nos revelan, como hemos visto, la construcción de dos cubos más que se citan en la documentación como "el cubo de la pólvora" y "el cubo postrero (último) que se hace en la fortaleza". Estas dos construcciones, cuyo fín en ese momento desconocemos, fueron levantadas entre 1541 y 1543 a la vez que se reformaba todo el interior del Cubo de Obras y Bosques para archivar los papeles ${ }^{58}$, posiblemente previniendo un crecimiento del espacio destinado a la guarda y custodia de la documentación de la Corona. Y para tal fin, se actúa en otros puntos de la fortaleza que necesitaban de reformas urgentes y que se llevan gran parte del presupuesto: las chapaduras de cantería, orientadas sobre todo a la zona externa del edificio, y el corredor del cuarto delantero de la fortaleza que daba al patio principal.

57 AGS, CMC, 1EP, 1380. Obras que se pagan con el dinero de las licencias de esclavos de las Indias. Así queda consignado en una Cédula Real de su Alteza el Príncipe Felipe a su contador Ochoa de Luyando fechada en Valladolid a 17 de octubre de 1543.

58 AGS, CMC, 1EP, 1410, s.f. Data de las obras a finales de 1543: "el cubo que se hazia para el archibo de mas de los otros dos cubos que estaban acabados". 


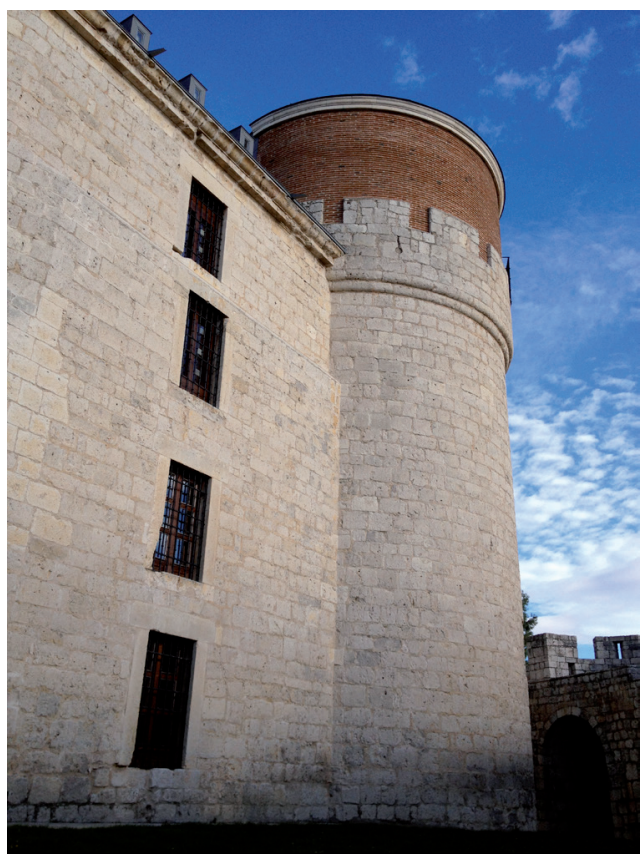

Fig. 3. Exterior del Cubo de Obras y Bosques, Archivo General de Simancas, c. $1541-$ 1543. Fotografía de la autora. Insertar acorde con su referencia, en la nota al pie 56.

Para las obras de la chapadura se gastó la suma de noventa y cinco mil ochocientos treinta y ocho maravedís ${ }^{59}$. Intervino el cantero Rodrigo de la Riva, que había trabajado años antes con Luis de Vega en otras obras vallisoletanas ${ }^{60}$, junto a Juan de Escalante y Rodrigo de la Maza. Para las obras del corredor delantero que "se caía", se gastaron ciento catorce mil cincuenta y un maravedís. El antiguo corredor en malas condiciones se derribó y se hizo de nuevo, interviniendo en la obra Francisco de Salamanca, un artífice que también trabaja en el Alcázar de Madrid $^{61}$ y para las reformas ulteriores de la fortaleza simanquina ya que es a él a quien Diego de Ayala y Felipe II encargan las primeras trazas del proyecto de ampliación de la misma en el año $1572^{62}$. Se interviene, a su vez, en la capilla que ya existía en el edificio con trabajos de yesería y carpintería ${ }^{63}$;

59 AGS, CMC, 1EP, 1410, s.f. Cuentas de Hernaldo Bernaldo, año 1542.

60 Rodrigo de la Riva trabaja en 1534 en la Casa del Comendador Rodrigo Enríquez, en Valladolid, bajo las condiciones de Luis de Vega. Se conservan a su vez, documentos que prueban su intervención en la Casa del Capitán Don Andrés de Herrera, cuyas condiciones y traza están atribuídas también al Maestro Mayor de Obras Reales: URREA, J., Arquitectura y Nobleza. Casas y Palacios en Valladolid. Valladolid, Consorcio IV centenario de la ciudad de Valladolid, 1996.

61 AGS, CMC, 1EP, 522, 1558. Cuentas de Alonso Hurtado, año 1546.

62 BUSTAMANTE, A., op. cit., p. 160. Su muerte en 1573 dio paso a su hijo Juan de Salamanca que ocupó su lugar tanto en las reformas de Simancas como en la gran obra de reconstrucción de la ciudad de Valladolid tras el incendio de 1561 .

63 La capilla es una de las partes conservadas más importantes de la primitiva construcción, en gran parte gracias a la bóveda que la cubre, ejemplar bellísimo propio de la segunda mitad del siglo XV, con 
en el cuarto real, sobre todo en labores relacionadas con acabados, puertas y ventanas que se encontraban en mal estado; en una cocina que se lleva a cabo ya en $1545^{64}$, y se trabaja en la ampliación de otras zonas como la plaza que se creó frente al archivo, una calle que llevara directa a la iglesia desde el mismo $^{65}$ o el puente "para venyr a un myrador de la dicha fortaleza", para las que se gastaron quinientos ducados pagados con el dinero de las licencias de esclavos de las Indias ${ }^{66}$. En la Ilustración 4 que acompaña a estas líneas se puede apreciar sobre la planta del Archivo las zonas que fueron intervenidas en el siglo $\mathrm{XVI}^{67}$.

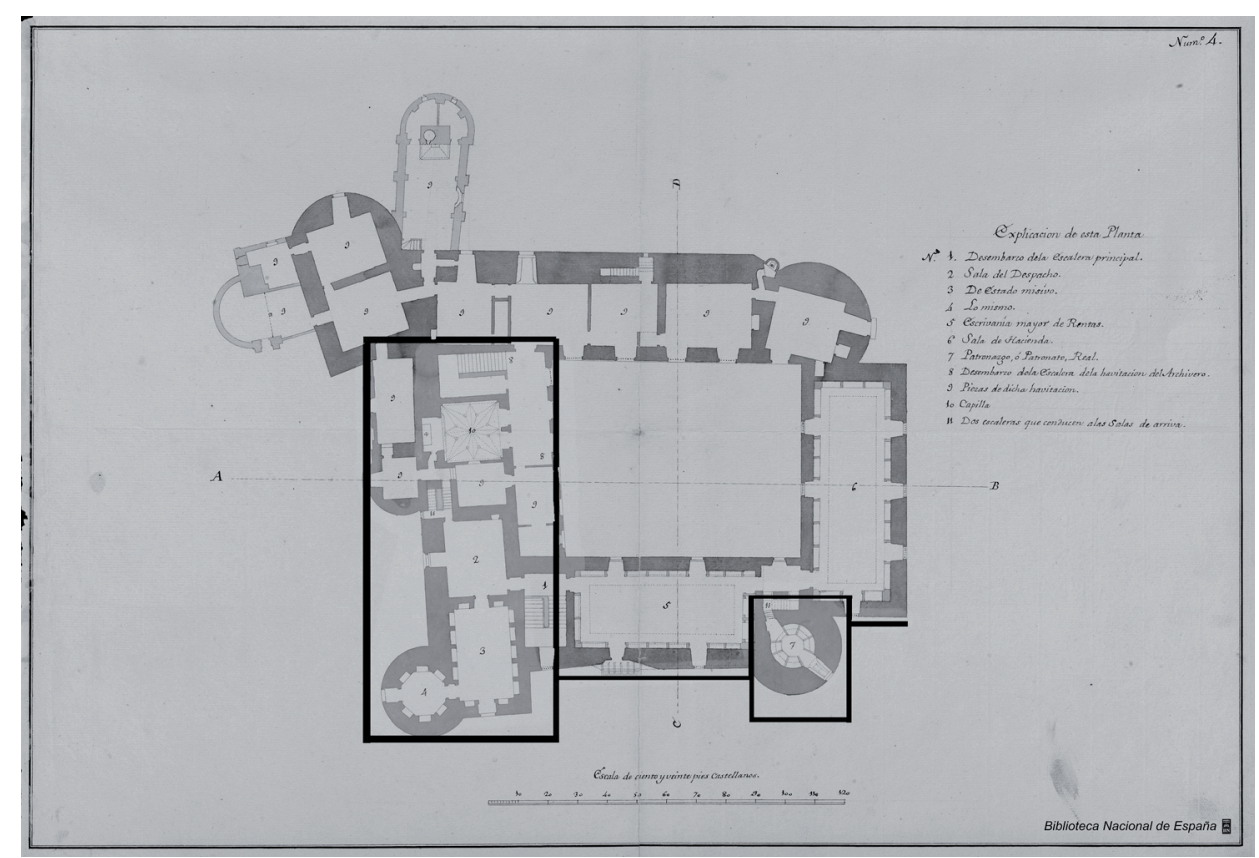

Fig. 4. VENTURA RODRIGUEZ - Proyecto para el Archivo Real de Simancas (con marcas de la autora en las partes de la fortaleza en las que se interviene en el siglo XVI), 1762. Biblioteca Nacional de España, Madrid. Fuente: Colección Online Arte Biblioteca Nacional de España. Insertar acorde con su referencia, en la nota al pie 67.

nueve escudos que la ornamentan: uno en el centro de mayor tamaño con las armas de los Almirantes y ocho alrededor, cuatro iguales en diseño al central y cuatro de los Velasco con el emblema de castillos y leones. Son el testimonio parlante de los edificadores de la capilla, el Almirante Don Alonso Enríquez y su esposa Doña María de Velasco.

64 AGS, CMC, 1EP, 1318, 1339. Cuentas del Bachiller Carrasco, año 1545.

65 AGS, CCA, CEDULAS, 111, FOL. 73; AGS, CCA, CEDULAS, 112, FOL. 9-10. Cédulas Reales de 30 de Noviembre de 1544 y 28 de Marzo de 1545, para que se le paguen a Juan Mosquera de Molina el importe gastado a su costa en estas obras.

66 AGS, ESTADO, LEG. 21, FOL. 192.

67 Ilustración 4. Proyecto para eñ Archivo Real de Simancas, del arquitecto Ventura Rodríguez, fechado en 1762. Pluma sobre papel, propiedad de la Biblioteca Nacional de España, Madrid. Sobre esta planta (de época posterior) podemos ver las zonas sobre las que se trabajó en época de Francisco de los Cobos. 
Estos datos nos muestran como estas obras, aparte de ser obviamente el germen del archivo de Simancas que conocemos en la actualidad, fueron de gran envergadura acorde con el plan ideado por el secretario imperial Francisco de los Cobos para la colocación de los documentos de la corona en este enclave. La historiografía dedicada al Archivo General de Simancas no había prestado especial atención a estas obras, reduciéndolas casi por unanimidad a la llevada de papeles al Cubo de Patronazgo Real y su consiguiente reforma interior basada en los distintos pisos de estanterías citados y descritos. La fortaleza de Simancas tras las obras trazadas, según parece, por Luis de Vega, se convierte en un edificio acorde con el gusto del momento que alberga las condiciones necesarias para el fín impuesto por el monarca hasta que pasadas unas décadas, se amplía y reforma tras las sucesivas peticiones del archivero Diego de Ayala que estaban justificadas por la masiva llegada de documentación ${ }^{68}$, ya durante el reinado de Felipe II.

\section{Documentos}

Se aportan a continuación una reducida selección de las fuentes primarias localizadas sobre las obras que se realizan en la fortaleza de Simancas entre los años 1539 y 1545. Dado que la mayor parte de la documentación aportada en el presente trabajo pertenece a los fondos de Contadurías Mayores de Cuentas del Archivo General de Simancas y son las propias cuentas del alcaide de la fortaleza, la transcripción de los mismos en un artículo de estas características es impensable debido a su envergadura y se han trascrito en parte a lo largo del presente trabajo, por lo que se transcriben aquí otros documentos de interés mencionados en el texto. Proceden del Archivo General de Simancas y son todos ellos inéditos. No obstante, las referencias archivísticas a esta documentación inédita citada, proveniente de las Contadurías Mayores de Cuentas, son las siguientes:

1. AGS, CONTADURÍA MAYOR DE CUENTAS, 1EP, 1410.

- Cargo y Data de las obras de la Fortaleza de Simancas. Hernando Bernaldo, alcaide de la Fortaleza. Febrero de 1540 a Agosto de 1544.

2. AGS, CONTADURÍA MAYOR DE CUENTAS, 1EP, 1318.

- Cargo y Data de la Fortaleza de Simancas. Bachiller Carrasco. 1544 y 1546.

- Copia de Carta de Francisco de los Cobos a Hernando Bernaldo, alcaide de la fortaleza de Simancas, sobre diversos temas tocantes a su casa en Valladolid y la fortaleza simanquina, en la que se menciona el memorial de Luis de Vega que se ha de seguir en las obras. Madrid, 24 de Febrero de 1540.

La relación de documentos transcritos sigue un orden cronológico de acuerdo a la fecha de creación. Como se explica por la descripción de cada uno de ellos, se trata de cartas enviadas por el Comendador Mayor de León Francisco de los Cobos a Juan

68 Una ampliación sobre la que no vamos a tratar aquí ya que ha sido abordada por la historiografía hasta la fecha. A destacar, entre otros: DE LA PLAZA BORES, A., op.cit. y BUSTAMANTE GARCÍA, A., op. cit. 
Mosquera de Molina y a Hernando Bernaldo, alcaide de la fortaleza de Simancas, y sus fechas extremas van de 1539 a 1543.

Las transcripciones respetan la forma original del texto, aunque se han incluído algunos signos de puntuación para facilitar la lectura y prescindimos de las abreviaturas con el mismo fín.

1. AGS, CMC, 1EP, Leg. 1410, s.f.

Carta de Francisco de los Cobos a Juan Mosquera de Molina, alcaide de la fortaleza de Simancas, sobre diversos temas tocantes a las primeras reformas del edificio.

Madrid, 29 de Octubre de 1539.

Muy virtuoso y comendador hermano. Luego a la ora que esta recivays entended en que con toda diligencia y presteza se adoben las puertas y ventanas de la fortaleza de Simancas de la manera que se puso en la tasacion que me enbiastes y en que se pongan en ella las cerraduras y candados que fueren menester de arte que queden estas cosas como deven estar y se haga lo de las puertas y ventanas y garitas en los ocho mill maravedis que concertastes o en menos si se pudiere y apuntareys aparte lo que costare ynbiareys la cuenta dello en siendo acabado y mira que se haga todo antes de diez de nobiembre porque asi conbiene y tanbien hazed poner en la fortaleza el trigo y cebada de la renta de Velliça y entregadlo a la persona que tiene cargo della Andrés de Prada para que este alli. Las otras cosas de reparos que vimos en la tasacion adelante se probeera como se haga. Nuestro señor vuestra muy vistuosa persona y casa grande y aquí como deseays. De Madrid a XXIX de octubre de 1539.

[Firmado] Covos Comendador Mayor

2. AGS, CMC, 1EP, Leg. 1410, s.f.

Carta de Francisco de los Cobos a Hernando Bernaldo, alcaide de la fortaleza de Simancas, sobre diversos temas tocantes a su casa en Valladolid y la fortaleza simanquina, en la que se menciona el memorial de Luis de Vega que se ha de seguir en las obras.

Madrid, 24 de Febrero de 1540.

Muy virtuoso señor. Recibi vuestra carta de X de febrero y he olgado mucho de saber que vos y la señora esteys buenos plega a dio que muchos años sea assi.

(...)

Por la necesidad que hay de los reparos en la fortaleza de Simancas scrivo al señor mosquera qye luego se junte con vos proveays en que se haga los reparos y provea las otras cosas que para la fortaleza son necesarias como mas largo vereis por su carta y le embio un memorial que Luys de Vega ha dexado de las condiciones con que le parece que se debe dar a destajo aquellos reparos de la chapadura de la cava y otros asuntos cuyo traslado yra aquí con esta y assimismo os embio una carta que Luys de Vega os scrive sobre ello vos os juntareys luego con el señor mosquera y entendereys en que aquello se haga lo mejor y mas barato y con mas brevedad que se pueda. Llevaos para ello ochocientos ducados haveislos de gastar assi en lo que concertardes los destajos como en el solar de la casa y empedrar el patio y adereçar las chimeneas y otras cosas de que hay necesidad de reparo según y como lo ordenare y señalare el comendador mosquera. Lo qual haveis de gastar por ante scrivano y con firmas del dicho comendador haveis de trabajar que todo se cumpla con hasta mill ducados o poco mas porque aunque me scrive el señor moquera que se hara con mill 
y doszientos ducados a my me dizen que bastaran hasta myll. Bien creo que por el señor mosquera no quedara de grangearlo todo tambien que no haya falta y que vos hareys lo mismo y assy os lo encomyendo y que de todo me aviseys particularmente. (...)

De madrid XXIIII de hebrero de DXL.

[Firmado] Covos comendador mayor.

3. AGS, CMC, 1EP, Leg. 1410, s.f.

Carta de Francisco de los Cobos a Hernando Bernaldo, alcaide de la fortaleza de Simancas, en la que se da noticia de la finalización de los dos primeros pisos del archivo. Madrid, 16 de Enero de 1543.

Muy virtuoso señor. El señor mosquera me ha scripto como esta acabada la obra del archivo de los dos suelos primero y segundo del cubo y que en lo alto del ay buena dispusicion para continuarse con los dineros que restan en vuestro poder que dize que seran hasta doszientos ducados poco mas o menos, yo le respondo que me paresce bien que se gasten en ella porque todo sera menester para las scripturas, vos le dexare trazar y hazer la obra como a el le pareciere sin meteros en esto mas de en pagar a los oficiales y obreros que en ello travajaren y los materiales que se compraren para la obra por sus libranzas como se suele hazer mirando que en el gasto dello aya buen recabdo.

(...)

De madrid a XVI de Enero de 1543.

[Firmado] Covos comendador mayor. 